\title{
Evidence for dark excitons in a single carbon nanotube due to the Aharonov-Bohm effect
}

\section{$\operatorname{AUTHOR}(\mathrm{S}):$}

Matsunaga, Ryusuke; Matsuda, Kazunari;

Yoshihiko, Kanemitsu

\section{CITATION:}

Matsunaga, Ryusuke ... [et al]. Evidence for dark excitons in a single carbon nanotube due to the Aharonov-Bohm effect. Physical Review Letters 2008, 101(14): 147404.

\section{ISSUE DATE:}

2008-10

URL:

http://hdl.handle.net/2433/87345

RIGHT: 


\title{
Evidence for Dark Excitons in a Single Carbon Nanotube due to the Aharonov-Bohm Effect
}

\author{
Ryusuke Matsunaga, ${ }^{1}$ Kazunari Matsuda, ${ }^{1, *}$ and Yoshihiko Kanemitsu ${ }^{1,2,+}$ \\ ${ }^{1}$ Institute for Chemical Research, Kyoto University, Uji, Kyoto 611-0011, Japan \\ ${ }^{2}$ Photonics and Electronics Science and Engineering Center, Kyoto University, Kyoto 615-8510, Japan
}

(Received 21 March 2008; published 3 October 2008)

\begin{abstract}
We studied exciton structures and the Aharonov-Bohm effect in a single carbon nanotube using microphotoluminescence (PL) spectroscopy under a magnetic field at low temperatures. A single sharp PL peak from the bright exciton state of a single carbon nanotube was observed under zero magnetic field, and the additional PL of dark exciton state appeared below the bright exciton peak under high magnetic fields. It was found that the split between the bright and dark exciton states is several millielectron volts at zero field. The tube diameter dependence of the splitting arises from the intervalley short-range Coulomb interaction.
\end{abstract}

PACS numbers: 78.67.Ch, 71.35.Ji, 78.55.-m

The electronic properties of single-walled carbon nanotubes (SWNTs) have attracted a great deal of attention [1] because of their degenerated band structures in $k$-space and enhanced Coulomb interaction in a quasi-one-dimensional (1D) system with a cylindrical structure [2]. The enhanced Coulomb interaction leads to the formation of stable 1D excitons with large binding energies [3,4]. Very recently, many theoretical studies have examined exciton structures [5-8] as the electron and hole spins, together with two degenerate $K$ and $K^{\prime}$ valleys, lead to multiple exciton states near the lowest energy $[9,10]$. The optical transition from the singlet exciton state with an odd parity and zero angular momentum to the ground state is dipole-allowed (bright), while the others are optically forbidden (dark). The nature of the dark exciton states in SWNTs is very different from that in IV and II-VI semiconductors [11,12]. The exciton fine structures including the energy separation between the bright and dark exciton states should be elucidated experimentally.

Magnetic fields modulate the phase of the electron wave functions in cylinder structures through the AharonovBohm effect. Ajiki and Ando theoretically predicted that the energies of the excitons in SWNTs are modulated directly by the magnetic flux threading the tube $[13,14]$. Recent ensemble-averaged absorption and photoluminescence (PL) spectroscopy experiments involving SWNTs have shown the spectral changes under high magnetic fields [15-18]. However, their optical spectra show multiple inhomogeneously broadened peaks with about $30 \mathrm{meV}$ linewidths. Furthermore, each carbon nanotube is aligned randomly in the sample with respect to the magnetic flux. Therefore, the spectral splitting has been observed only in extremely high magnetic fields $(>30 \mathrm{~T})$ $[15,16]$ and it is difficult to determine the exciton fine structures for zero magnetic field. Consequently, single carbon nanotube spectroscopy under a magnetic field is needed to avoid the sample inhomogeneity and to obtain information about the intrinsic excitonic properties of carbon nanotubes.
In this Letter, we report single carbon nanotube PL spectroscopy under magnetic fields up to $7 \mathrm{~T}$ to study the exciton fine structures in SWNTs. A new PL peak appeared below the bright exciton state under a magnetic field in the Voigt geometry. This PL peak resulted from the dark exciton due to the Aharonov-Bohm effect in a single carbon nanotube. We directly determined the splitting energy between the bright and dark exciton states, and found that it depends on the nanotube diameter.

The samples used in this study were spatially isolated carbon nanotubes synthesized on Si substrates using an alcohol catalytic chemical vapor deposition method [19]. The Si substrates were patterned with parallel grooves, typically about $1 \mu \mathrm{m}$ wide and $500 \mathrm{~nm}$ deep using an electron-beam lithography technique. The isolated carbon nanotubes grow from one side toward the opposite side of the groove. Several different samples were prepared by changing the growth temperature.

Magneto-PL measurements of a single carbon nanotube were performed using a home-built variable-temperature confocal microscope. The samples were mounted in a cryostat under a superconducting magnet with fields up to $7 \mathrm{~T}$. The excitation light from a He-Ne laser $(1.96 \mathrm{eV})$ was focused on the sample surface through a microscope objective to detect PL signals from a single carbon nanotube. The polarization direction of the excitation laser was controlled by rotating a $\lambda / 2$ wave plate. The PL signal from a single carbon nanotube was detected by a $30-\mathrm{cm}$ spectrometer equipped with a liquid-nitrogencooled InGaAs photodiode array with a spectral range of 0.78 to $1.38 \mathrm{eV}$. The spectral resolution of the system was about $1.4 \mathrm{meV}$. Magneto-PL spectroscopy was carried out under Voigt geometry and Faraday geometry. In the Voigt geometry, in which laser light propagates perpendicular to the magnetic field, we can investigate the effect of the magnetic flux threading the nanotube axis, while in the Faraday geometry, in which the laser propagates parallel to the field, the magnetic flux is perpendicular to the tube axis. 
The solid line in Fig. 1(a) shows a typical PL spectrum of a single carbon nanotube suspended on the groove at about $40 \mathrm{~K}$ [assigned chiral index: $(7,6)$ ]. It is approximated by a Lorentzian function and its linewidth [fullwidth at half-maximum (FWHM) $\sim 3.4 \mathrm{meV}$ ] reflects homogeneous broadening $[20,21]$. The single sharp PL spectrum is in contrast to the multiple broad PL peaks in the ensemble-averaged spectrum (dotted line in Fig. 1(a)] of micelle-wrapped SWNTs dispersed in gelatin [22]. The isolated sharp spectrum of a single carbon nanotube enables us to observe the spectral changes in detail, even under a magnetic field of several $\mathrm{T}$.

Figure 1(b) shows a polar plot of the PL intensity of a typical single carbon nanotube versus the polarization direction of the excitation laser light. The PL intensity shows strong polarization dependence and oscillates with a duration of $180^{\circ}$. Since a $1 \mathrm{D}$ dipole moment exists, strong optical absorption occurs when the polarization of the excitation light parallels the nanotube axis. The PL intensity of a SWNT is expected to be proportional to $\cos ^{2} \theta$, where $\theta$ is the angle between the tube axis and the polarization of the excitation laser light [23-25]. This PL anisotropy is useful for determining the direction of the observed carbon nanotube. Therefore, we can consider the relative angle $\alpha$ between the tube axis and magnetic field in the Voigt geometry [Fig. 1(c)].

Figure 2(a) shows the normalized PL spectra of a single $(9,4)$ carbon nanotube under a magnetic field in the Voigt geometry $\left(\alpha \approx 9^{\circ}\right)$. A single sharp PL spectrum arising
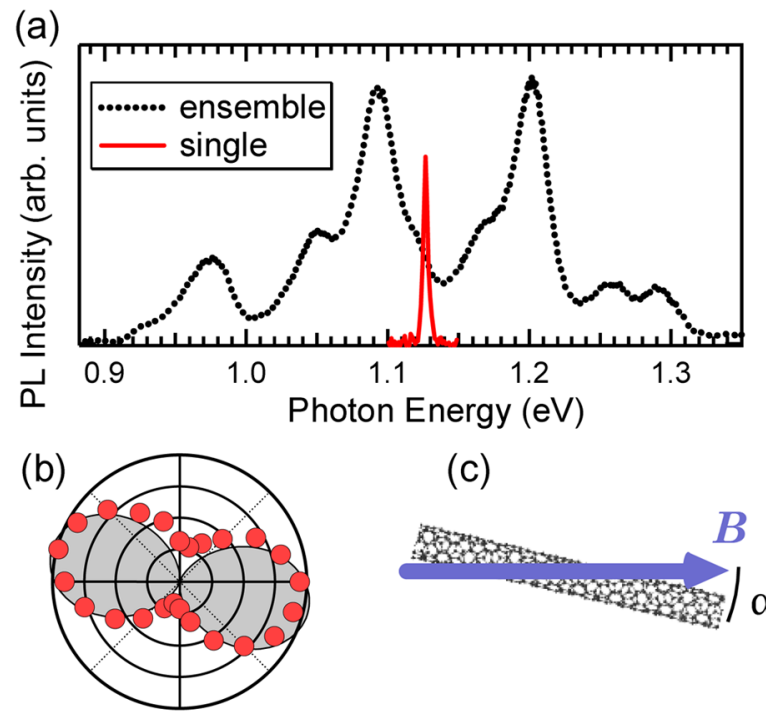

(c)

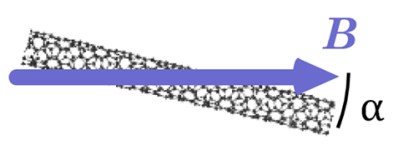

FIG. 1 (color online). (a) PL spectrum of a typical suspended single SWNT [assigned chiral index $(7,6)$ ] in comparison with the ensemble-averaged spectrum of micelle-wrapped SWNTs dispersed in gelatin. (b) Polar plot of the PL intensity of a typical single SWNT versus the polarized direction $\theta$ of the excitation laser. The PL data (circle) were fitted using $\cos ^{2} \theta$ (solid line). (c) Schematic of the directional relationship between the nanotube axis and magnetic field. In this case, the relative angle $\alpha$ was estimated to be about $13^{\circ}$. from bright exciton recombination is observed at zero magnetic field. An additional peak is clearly observed below the bright exciton peak with an increasing field. These PL spectra are fit well by two Lorentzian functions to evaluate the energy positions of the two peaks. The lower energy peak shows a redshift and the intensity relative to the higher peak increases with the magnetic field. Figure 2(b) shows the results of similar experiments on a single $(9,5)$ nanotube performed in the Faraday geometry, where the magnetic flux is perpendicular to the nanotube axis. No spectral splitting induced by the magnetic field is observed in the Faraday geometry, even under a magnetic
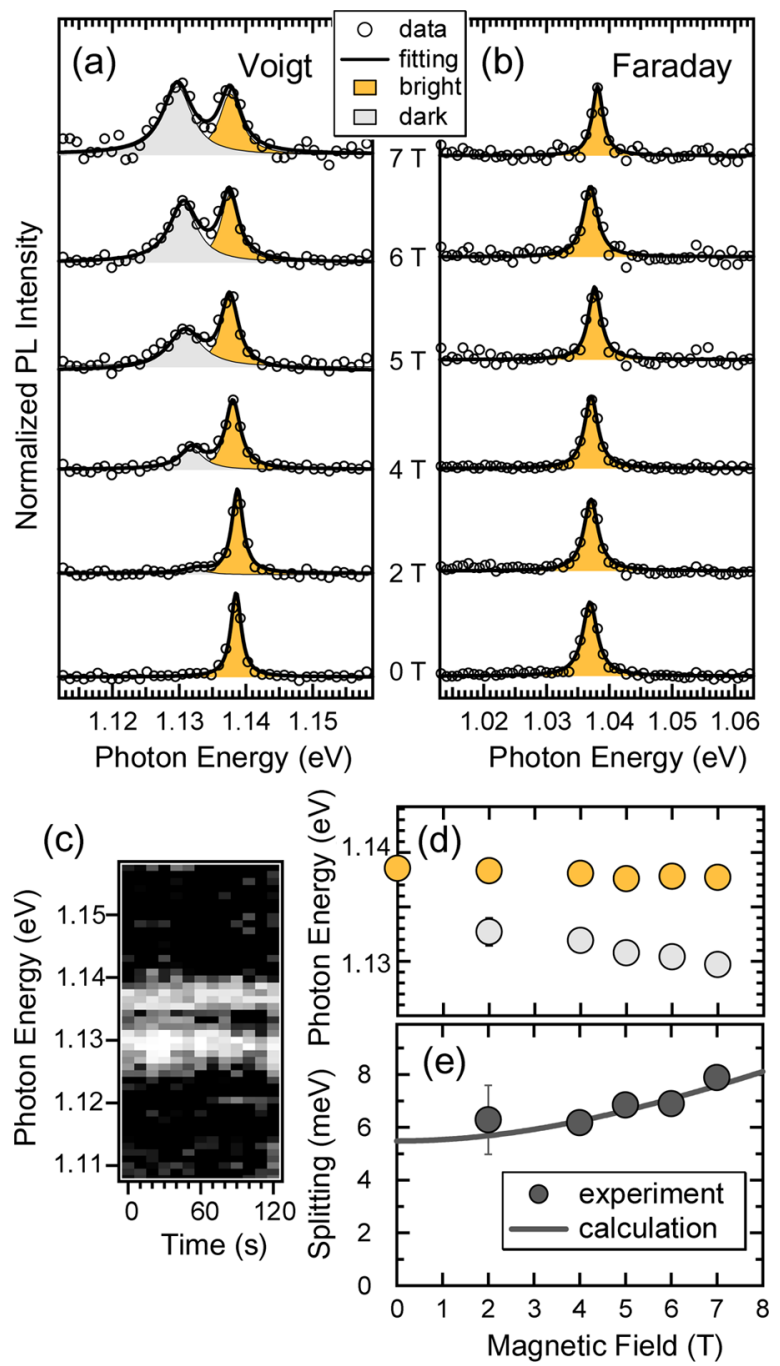

FIG. 2 (color online). (a) Normalized magneto-PL spectra of a single $(9,4)$ carbon nanotube at $20 \mathrm{~K}$ in the Voigt geometry $(\alpha \approx$ $\left.9^{\circ}\right)$. The split PL spectra are fit by two Lorentzian functions. (b) The normalized magneto-PL spectra of a single $(9,5)$ carbon nanotube at $20 \mathrm{~K}$ in the Faraday geometry. The PL spectra are fit by a Lorentzian function. (c) Temporal evolution of the PL spectra with $10 \mathrm{~s}$ accumulation time in (a) at $7 \mathrm{~T}$. (d) The energy positions of the bright and dark exciton states as a function of the magnetic field in (a). (e) Splitting energy $\Delta_{b d}(B)$ versus the magnetic field. The solid line corresponds to the theoretically fitted curve based on Eq. (2). 
field of $7 \mathrm{~T}$. Note that the splitting of the PL peak occurs due to the magnetic flux parallel to the nanotube axis. Figure 2(c) shows the temporal trace of the PL spectrum in Fig. 2(a) at $7 \mathrm{~T}$ with accumulation time of $10 \mathrm{~s}$. Two peaks clearly show a constant splitting with time evolution over $120 \mathrm{~s}$, which means that it is different from timedependent spectral fluctuation [26]. Our experimental result indicates that the observed PL peak splitting arises from the Aharonov-Bohm effect in a single carbon nanotube.

We now discuss the Aharonov-Bohm splitting observed in a single carbon nanotube. The effective magnetic flux threading the nanotube is experimentally evaluated as $\phi=$ $\left(\pi d^{2} / 4\right) B \cos \alpha$, where $d$ is the nanotube diameter and $B$ is the strength of the magnetic field. According to the theoretical calculation by Ajiki and Ando [13], the degenerated band-gaps in semiconducting carbon nanotubes at the $K$ and $K^{\prime}$ valleys are lifted due to the Aharonov-Bohm effect. The split between the two states, $\Delta_{A B}$, is described by

$$
\Delta_{A B}=6 E_{g} \phi / \phi_{0} \quad\left(\phi / \phi_{0} \leq 1 / 6\right),
$$

where $\phi_{0}=c h / e$ is the magnetic quantum and $E_{g}$ is the band-gap energy of the carbon nanotubes.

In the excitonic picture, two degenerated valleys create four singlet exciton states, $K K, K^{\prime} K^{\prime}, K K^{\prime}$, and $K^{\prime} K$, in which the $K K$ exciton consists of an electron (hole) in the $K(K)$ valley [14]. Moreover, the singlet exciton states with zero angular momentum, $K K$ and $K^{\prime} K^{\prime}$, split into the bonding and antibonding exciton states due to the shortrange Coulomb interaction. The bonding state is odd parity (bright) and the antibonding is even (dark) [14]. When the magnetic flux threads the nanotube axis, the exciton states at the $K$ and $K^{\prime}$ valleys become independent of each other, and the singlet dark state with even parity gradually brightens as the magnetic field is increased. Using Eq. (1), the energy difference $\Delta_{b d}(B)$ between the bright and dark exciton states under magnetic field can be described by

$$
\Delta_{b d}(B)=\sqrt{\Delta_{b d}^{2}+\Delta_{A B}^{2}(B)},
$$

where $\Delta_{b d}$ is the energy splitting at zero magnetic field [17].

Figure 2(d) shows the bright and dark exciton energies estimated from the Lorentzian spectra fitting procedure as a function of magnetic field in Fig. 2(a). The blueshift of the bright exciton peak is not experimentally observed up to $7 \mathrm{~T}$. Here, for the evaluation of $\Delta_{b d}$, we assumed that the Aharonov-Bohm splitting is proportional to the magnetic flux, that is, $\Delta_{A B}=\mu \phi$, where $\mu$ is a constant [17]. Figure 2(e) shows the experimentally observed splitting values and the theoretical curve based on Eq. (2) where $\Delta_{b d}$ and $\mu$ are fitting parameters. The theoretically calculated curves reproduce the experimental results well. Here the value of $\Delta_{b d}$ is about $5.5 \mathrm{meV}$ under zero magnetic field and the parameter $\mu$ is $1.1 \mathrm{meV} / \mathrm{Tnm}^{2}$ [27]. This result indicates that the dark exciton state exists about $5.5 \mathrm{meV}$ below the bright exciton state in the $(9,4)$ SWNT.
It is found that the bright exciton linewidth in the Voigt geometry becomes broad linearly with an increase of the field up to $7 \mathrm{~T}$, from $1.9 \mathrm{meV}$ at $0 \mathrm{~T}$ to $3.9 \mathrm{meV}$ at $7 \mathrm{~T}$ in Fig. 2(a), while that in the Faraday geometry remains unchanged. The PL linewidth is determined by the dephasing time of excitons $[20,21]$. Our observation suggests that the symmetry breaking of the exciton wave functions due to the Aharonov-Bohm effect causes the shortening of the dephasing time of bright excitons at low temperatures. It is pointed out that phonons cannot scatter excitons from the bright state (odd parity) to the dark state (even parity) without any symmetry breaking process $[7,18]$. As discussed before, the Aharonov-Bohm flux influences the parity of the dark exciton state, which allows the scattering from the bright to the dark states and results on the broadening of the bright exciton linewidth.

Magneto-PL spectroscopy was performed for many single carbon nanotubes at low temperatures in the Voigt geometry. Figure 3 shows several PL spectra obtained from single carbon nanotubes for different chiralities. The PL spectra under magnetic fields show splitting, or an additional new peak arising on the lower energy side of the bright exciton peak. The magneto-PL spectra are fitted by two Lorentzian functions. The energy splitting is dependent on the nanotube chirality. For some nanotubes, the PL spectral diffusion occurs [26]. The PL spectra shift to the lower or higher energy sides under the magnetic field, as

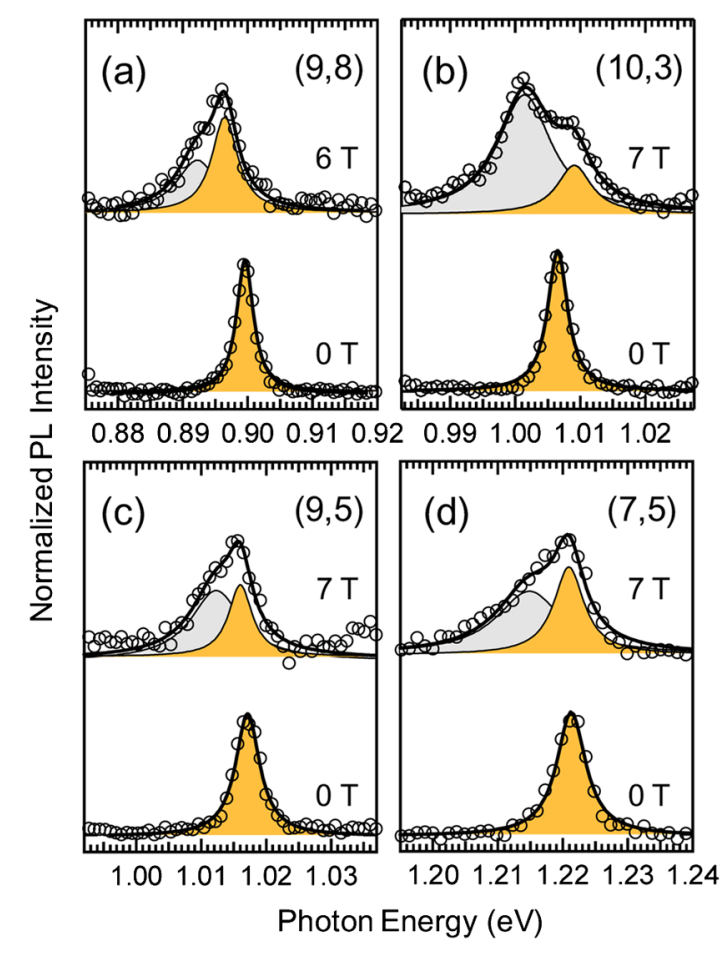

FIG. 3 (color online). Magneto-PL spectra obtained from typical single carbon nanotubes for different chiralities in the Voigt geometry. The chralities for (a)-(d) are $(9,8),(10,3),(9,5)$, and $(7,5)$, respectively. The relative angles $\alpha$ for (a)-(d) are about $14^{\circ}, 10^{\circ}, 63^{\circ}$, and $28^{\circ}$, respectively. 


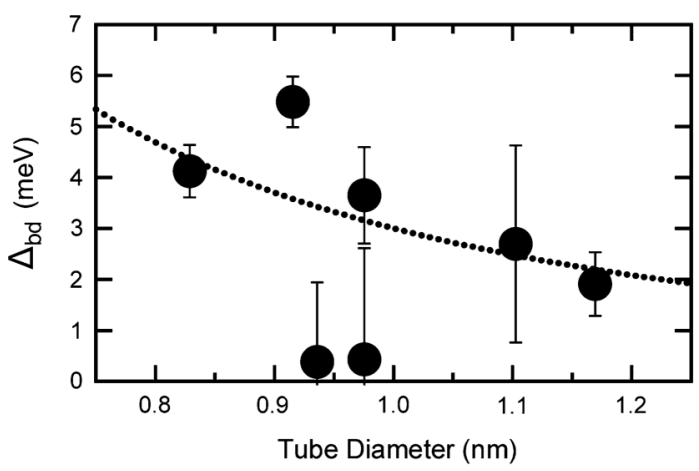

FIG. 4. Tube diameter dependence of the splitting energy between the bright and dark exciton states at zero field. The dotted line corresponds to the theoretically predicted dependence of $1 / d^{2}$.

shown in Fig. 3. This spectral shift suggests that some extrinsic effects, such as gas adsorption, would influence the optical properties of the single carbon nanotubes.

Here, we focus on the energy difference between the bright and dark exciton states to study the Aharonov-Bohm splitting. The bright-dark exciton splitting $\Delta_{b d}$ at zero field was estimated using the procedure described above [Eq. (2)]. Figure 4 shows the diameter dependence of $\Delta_{b d}$, which depends on the nanotube diameter and increases for smaller diameter tubes. This diameter dependence can be understood in terms of the short-range Coulomb interaction between the excitons at the $K$ and $K^{\prime}$ valleys [14]. The dotted line corresponds to the theoretically predicted dependence of $\Delta_{b d}$, which is proportional to $1 / d^{2}[8,14]$, although there is a large difference in the magnitude of $\Delta_{b d}$ between the experimental observation and the theoretical prediction [8]. This theoretical predicted diameter dependence can reproduce the experimental results.

In summary, we report direct evidence of dark excitons in a single carbon nanotube using magneto-micro-PL spectroscopy at low temperatures. The PL peak arising from the dark exciton state appeared below the bright exciton peak on increasing the magnetic field in the Voigt geometry. The spectral splitting between the dark and bright exciton states depends on the effective magnetic flux threading the nanotube. We observed the Aharonov-Bohm effect in a single carbon nanotube experimentally, and found that the dark exciton state was located several millielectron volts below the bright exciton state. The splitting depends strongly on the nanotube diameter and arises from the short-range Coulomb interaction.

The authors thank Dr. S. Kasai and Professor T. Ono for technical support in fabricating the grooved Si substrates for SWNT growth and Dr. Y. Miyauchi and Prof. S. Maruyama for sample preparation of micelle-wrapped SWNTs dispersed in gelatin. This study was supported by a Grant-in-Aid for Scientific Research from JSPS, the Casio Foundation and Kyoto University G-COE program from MEXT. *matsuda@scl.kyoto-u.ac.jp

${ }^{+}$kanemitu@scl.kyoto-u.ac.jp

[1] R. Saito, G. Dresselhaus, and M. S. Dresselhaus, Physical Properties of Carbon Nanotubes (Imperial College Press, London, 1998).

[2] T. Ando, J. Phys. Soc. Jpn. 66, 1066 (1997).

[3] T. Ogawa and T. Takagahara, Phys. Rev. B 43, 14325 (1991).

[4] F. Wang, G. Dukovic, L. E. Brus, and T. F. Heinz, Science 308, 838 (2005).

[5] V. Perebeinos, J. Tersoff, and Ph. Avouris, Phys. Rev. Lett. 92, 257402 (2004).

[6] H. Zhao and S. Mazumdar, Phys. Rev. Lett. 93, 157402 (2004).

[7] V. Perebeinos, J. Tersoff, and Ph. Avouris, Nano Lett. 5, 2495 (2005).

[8] C.D. Spataru, S. Ismail-Beigi, R. B. Capaz, and S. G. Louie, Phys. Rev. Lett. 95, 247402 (2005).

[9] O. Kiowski, K. Arnold, S. Lebedkin, F. Hennrich, and M. M. Kappes, Phys. Rev. Lett. 99, 237402 (2007).

[10] H. Kishida, Y. Nagasawa, S. Imamura, and A. Nakamura, Phys. Rev. Lett. 100, 097401 (2008).

[11] P. D. J. Calcott, K. J. Nash, L. T. Canham, M. J. Kane, and D. Brumhead, J. Phys. Condens. Matter 5, L91 (1993).

[12] M. Nirmal, D. J. Norris, M. Kuno, M. G. Bawendi, Al. L. Efros, and M. Rosen, Phys. Rev. Lett. 75, 3728 (1995).

[13] H. Ajiki and T. Ando, J. Phys. Soc. Jpn. 62, 1255 (1993).

[14] T. Ando, J. Phys. Soc. Jpn. 75, 024707 (2006).

[15] S. Zaric, G. N. Ostojic, J. Kono, J. Shaver, V. C. Moore, M. S. Strano, R. H. Hauge, R. E. Smalley, and X. Wei, Science 304, 1129 (2004).

[16] S. Zaric, G. N. Ostojic, J. Shaver, J. Kono, O. Portugall, P. H. Frings, G. L. J. A. Rikken, M. Furis, S. A. Crooker, X. Wei, V. C. Moore, R. H. Hauge, and R. E. Smalley, Phys. Rev. Lett. 96, 016406 (2006).

[17] J. Shaver, J. Kono, O. Portugall, V. Krstic, G. L. J. A. Rikken, Y. Miyauchi, S. Maruyama, and V. Perebeinos, Nano Lett. 7, 1851 (2007).

[18] I. B. Mortimer and R. J. Nicholas, Phys. Rev. Lett. 98, 027404 (2007).

[19] S. Maruyama, R. Kojima, Y. Miyauchi, S. Chiashi, and M. Kohno, Chem. Phys. Lett. 360, 229 (2002).

[20] T. Inoue, K. Matsuda, Y. Murakami, S. Maruyama, and Y. Kanemitsu, Phys. Rev. B 73, 233401 (2006).

[21] K. Matsuda, T. Inoue, Y. Murakami, S. Maruyama, and Y. Kanemitsu, Phys. Rev. B 77, 033406 (2008).

[22] H. Hirori, K. Matsuda, Y. Miyauchi, S. Maruyama, and Y. Kanemitsu, Phys. Rev. Lett. 97, 257401 (2006).

[23] A. Hartschuh, H.N. Pedrosa, L. Novotny, and T.D. Krauss, Science 301, 1354 (2003).

[24] J. Lefebvre, J. M. Fraser, P. Finnie, and Y. Homma, Phys. Rev. B 69, 075403 (2004).

[25] D. E. Milkie, C. Staii, S. Paulson, E. Hindman, A. T. Johnson, and J.M. Kikkawa, Nano Lett. 5, 1135 (2005).

[26] K. Matsuda, T. Inoue, Y. Murakami, S. Maruyama, and Y. Kanemitsu, Phys. Rev. B 77, 193405 (2008).

[27] The value of $\mu$ is close to but a little smaller than the predicted value $1.6 \mathrm{meV} / \mathrm{T} \mathrm{nm}^{2}$ from Eq. (1). 